\title{
Identification of a Novel HIV-1 Second-Generation Recombinant Form (CRF01_AE/CRF07_BC) in Jilin, China
}

\author{
Yi Feng, ${ }^{1, *}$ Xingguang Li, ${ }^{1,2, *}$ Xihui Zang, ${ }^{3}$ Qi Guo, ${ }^{3}$ Liuyan Sun, ${ }^{3}$ Xiang He, ${ }^{1,4}$ Hui Xing, and Yiming Shao ${ }^{1}$
}

\begin{abstract}
We report here a novel HIV-1 second-generation recombinant form (CRF01_AE/CRF07_BC) isolated from an HIV-positive male subject infected through heterosexual contact in Jilin province in northeastern China. Phylogenic analysis shows that this novel second-generation recombinant (JL.RF09) was composed of two well-established circulating recombinant forms (CRF01_AE and CRF07_BC), with two recombinant breakpoints observed in the vpu/env and env gene regions, respectively. The CRF01_AE region of the recombinant was clustered together with a previously described subcluster 4a lineage of CRF01_AE, which is exclusively circulating among men who have sex with men (MSM) in northern China, indicating that the parental origin of the CRF01_AE region in JL.RF09 was from MSM in north China. The CRF07_BC regions of the recombinant are clustered within the CRF07_BC cluster but are distinct from other CRF07_BC references. The detailed origin of CRF07_BC in this recombinant is still not clear. The emergence of the novel HIV-1 recombinant indicates the ongoing generation of recombinants involving CRF01_AE and CRF07_BC, and may provide vital insights into our understanding of the dynamics and complexity of the HIV-1 epidemic in China.
\end{abstract}

$\mathbf{T}$ HE EXTREMELY HIGH LEVEL of genetic variation of human immunodeficiency virus type 1 (HIV-1) is one of its important features. In addition to a naturally high mutation rate, HIV-1 also generates new variants through genetic recombination when a patient has been coinfected with two or more strains. Based on the phylogenetic analysis of viral genome sequences, the HIV-1 strains circulating globally are currently categorized into four groups: $\mathrm{M}, \mathrm{O}, \mathrm{N}$, and P. HIV-1 group $M$ strains play a major role in the global HIV-1 epidemic and are further divided into nine subtypes (A, B, C, D, $\mathrm{F}, \mathrm{G}, \mathrm{H}, \mathrm{J}$, and $\mathrm{K}$ ) and two sets of subsubtypes (A1, A2 and $\mathrm{F} 1, \mathrm{~F} 2){ }^{1}$ In addition, an increasing number of circulating recombinant forms (CRFs) and numerous unique recombinant forms (URFs) have been reported, ${ }^{1}$ especially in regions where multiple subtypes/subsubtypes and CRFs of HIV-1 cocirculated in the same high-risk population. On the other hand, the emergence of a large number of novel HIV-1 CRFs and URFs indicated that multiple genotypes of HIV-1 are cocirculating in the same risk population and more people have been coinfected with different strains. ${ }^{2}$
In the past 20 years, many HIV-1 strains with different genotypes and many different patterns of recombinant forms have been found in China. The predominant HIV-1 genotypes in China are subtype B' (the Thailand variant of subtype B), CRF01_AE, CRF07_BC, and CRF08_BC. These four genotypes constituted $92.8 \%$ of HIV-1 infections in China according to the national cross-sectional study of HIV molecular epidemiology in $2006 .{ }^{3}$ It has been concluded that, in China, CRF01_AE strains are mainly transmitted among injection drug users (IDUs) and sexually promiscuous groups in southern China, ${ }^{4,5}$ and CRF07_BC strains are predominantly in IDU populations in western China. ${ }^{6,7}$

However, in recent years, these two genotypes of strains began to expand to almost the entire country, and have been detected in various high-risk populations including IDUs, heterosexuals, and men having sex with men (MSM). ${ }^{3,8} \mathrm{Co}$ circulation of the CRF01_AE and CRF07_BC strains creates opportunities for the emergence of new, second-generation recombinants. To date, three different URFs composed of CRF01_AE and CRF07_BC have been reported in China. ${ }^{9-11}$

\footnotetext{
${ }^{1}$ State Key Laboratory for Infectious Disease Prevention and Control, National Center for AIDS/STD Control and Prevention, Chinese Center for Disease Control and Prevention, Collaborative Innovation Center for Diagnosis and Treatment of Infectious Diseases, Beijing, China.

${ }^{2}$ HIV Molecular Epidemiology and Virology Research Group, The State Key Laboratory of Virology, Wuhan Institute of Virology, University of Chinese Academy of Sciences, Wuhan, China.

${ }^{3}$ Jilin Provincial Center for Disease Control and Prevention, Jilin, China.

${ }^{4}$ Guangdong Provincial Institute of Public Health, Guangdong, China.

*These authors contributed equally to this work.
} 
In the present study, we detected a novel HIV-1 secondgeneration recombinant form (CRF01_AE/CRF07_BC), designated as JL.RF09, by near full-length genome (NFLG) analyses, isolated from an HIV-positive consenting male subject among the heterosexual population in Jilin province of northeastern China, which is different from the CRF01_AE/ CRF07_BC recombinant viruses previously reported in China.

In this study, an HIV-1-positive plasma sample was collected from a 22-year-old male peasant (Patient JL.RF09) in Changchun city of Jilin province in northeastern China. JL.RF09 is a Chinese citizen of Han ethnicity, residing in Harbin city of Heilongjiang province neighboring Jilin province; he was diagnosed as HIV positive on May 28, 2010 when he had a physical examination as a detained person in Changchun city of Jilin province and was recruited from the Jilin Provincial Center for Disease Control and Prevention (JPCDC). A self-report by JL.RF09 shows that he was infected through heterosexual transmission. JL.RF09 was treatment naive for antiretroviral therapy before his blood samples were collected. His CD4 ${ }^{+}$ T cell count was 639 cells/ $\mu$ l on June 22, 2011 when the plasma was collected. Written informed consent was obtained from the subject prior to sample collection. The study was approved by the institutional review boards of the National Center for AIDS/ STD Control and Prevention of China.

The amplification and sequencing of the new generated NFLG were performed as previously described. ${ }^{11,12}$ The amplification primers were as follows: the upper primer 07_BC-FL1.5 (5'-CCTTGAGTGCTTCAAGTAGTGTGTG CCCGTCTGT-3', HXB2 nt 548 to 571), the lower primer 07_BC-FL1.3 (5'-ACTACTTGAAGCACTCAAGGCAAG CTTTATTG-3', HXB2 nt 9632 to 9611), the upper primer 07_BC-FL2.5 (5'-AGTAGTGTGTGCCCGTCTGTTGTGT GACTC-3', HXB2 nt 562 to 581), and the lower primer 07_BC-FL2.3 (5'-TGAAGCACTC AAGGCAAGCTTTA TTGAGGCTTA-3', HXB2 nt 9626 to 9604), for the first and second rounds of nested polymerase chain reaction (PCR) amplification, respectively. Recombination breakpoints were determined using SimPlot and jpHMM (http://jphmm.gobics .de/submission_hiv).

Additional reference sequences of CRF01_AE and CRF07_ $\mathrm{BC}$ (Cluster 1 to 7 of CRF01_AE based on a previous muti- lineage CRF01_AE study $^{13}$ and a new identified cluster of CRF07_BC mainly transmitted among MSMs in China ${ }^{14-17}$ ) were introduced to the subregion phylogenetic analysis to clarify the origin of the recombinant fragments. They are labeled as CRF01-1 through CRF01-7 and CRF07-1, respectively, in the subregion trees. Cluster 4 of CRF01_AE is further divided into subclusters $4 \mathrm{a}$ and $4 \mathrm{~b}$ lineage (designated as CRF01-4a and CRF01-4b) since it contains two subclusters. ${ }^{13}$ The NFLG sequence amplified from Patient JL.RF09 was 8,923 nucleotides (nt) in size and the blast search data show that no evidence of sample contamination was detected. In the neighbor-joining tree constructed from the NFLG alignment, JL.RF09 clustered with the CRF07_BC and CRF08_BC ref erence sequences (bootstrap value $87 \%$ ), but formed a distinct monophyletic branch distantly related to the CRF07_BC and CRF08_BC reference sequences (Fig. 1).

SimPlot analysis shows that the NFLG sequence of JL.RF09 was composed of CRF01_AE and CRF07_BC, with one region of CRF01_AE inserted into a CRF07_BC backbone (Fig. 2A). And bootscanning analysis of the NFLG sequence reveals that the two unique recombination breakpoints corresponded to HXB2 nucleotide positions 6,241 and 8,370 , located in the vpu/env and env gene regions, respectively, and divide the NFLG into three regions: region I (HXB2, 790-6,241), CRF07_BC; region II (HXB2, 6,2428,370), CRF01_AE; and region III (HXB2, 8,371-9,411) (Fig. 2B). Similar results were obtained using online software jpHMM-HIV (http://jphmm.gobics.de/submission_hiv). The final map results of the schematic structure are shown in Fig. 2C. The profile of the recombinant structure of JL.RF09 is distinct from that of the CRF01_AE/CRF07_BC recombinants previously reported in China. ${ }^{9-11}$

Subregion tree analysis of JL.RF09 further confirmed the two recombinant breakpoints identified by bootscanning analysis (Fig. 3). It also demonstrated that the CRF01_AE region (II) in JL.RF09 belonged to the CRF01_AE subcluster 4a lineage (designated CRF01-4a), which is exclusively circulating among MSM in northern China, ${ }^{13}$ with the support of a $100 \%$ bootstrap value, indicating that the parental origin of the CRF01_AE region (II) was from MSM-related CRF01_AE subcluster 4a lineage.

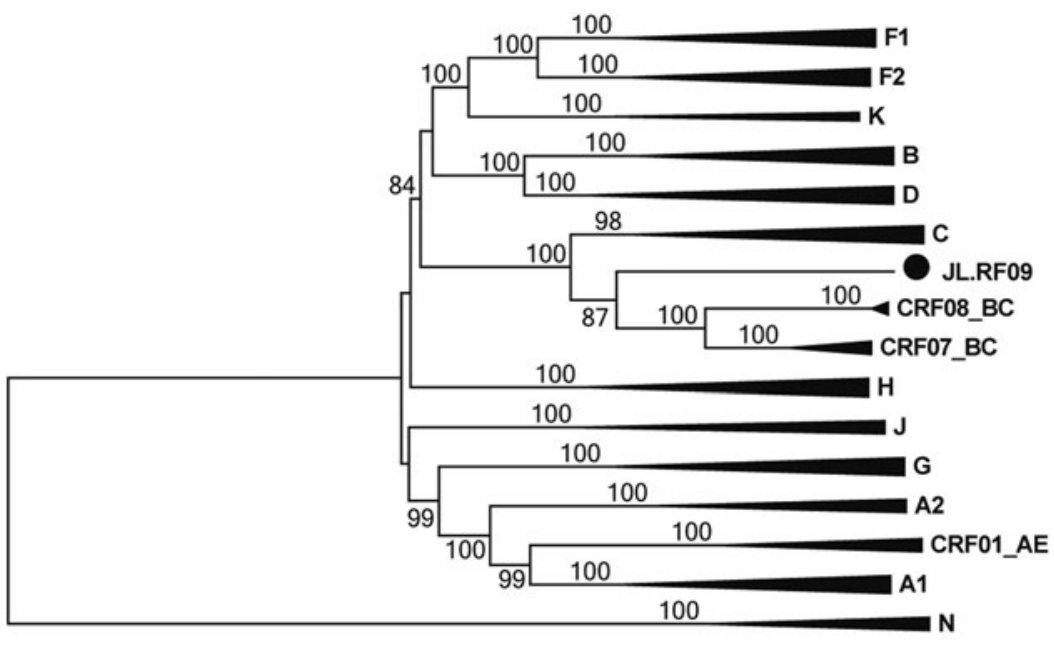

0.05
FIG. 1. A neighbor-joining phylogenetic tree of JL.RF09 with reference sequences constructed using MEGA 5.05. Solid circle (O) marks JL.RF09 throughout the article. The stability of each node was assessed by bootstrap analyses with 1,000 replicates and only significant bootstrap values of $70 \%$ or greater are shown at the corresponding nodes. The scale bar represents $5 \%$ genetic distance. 

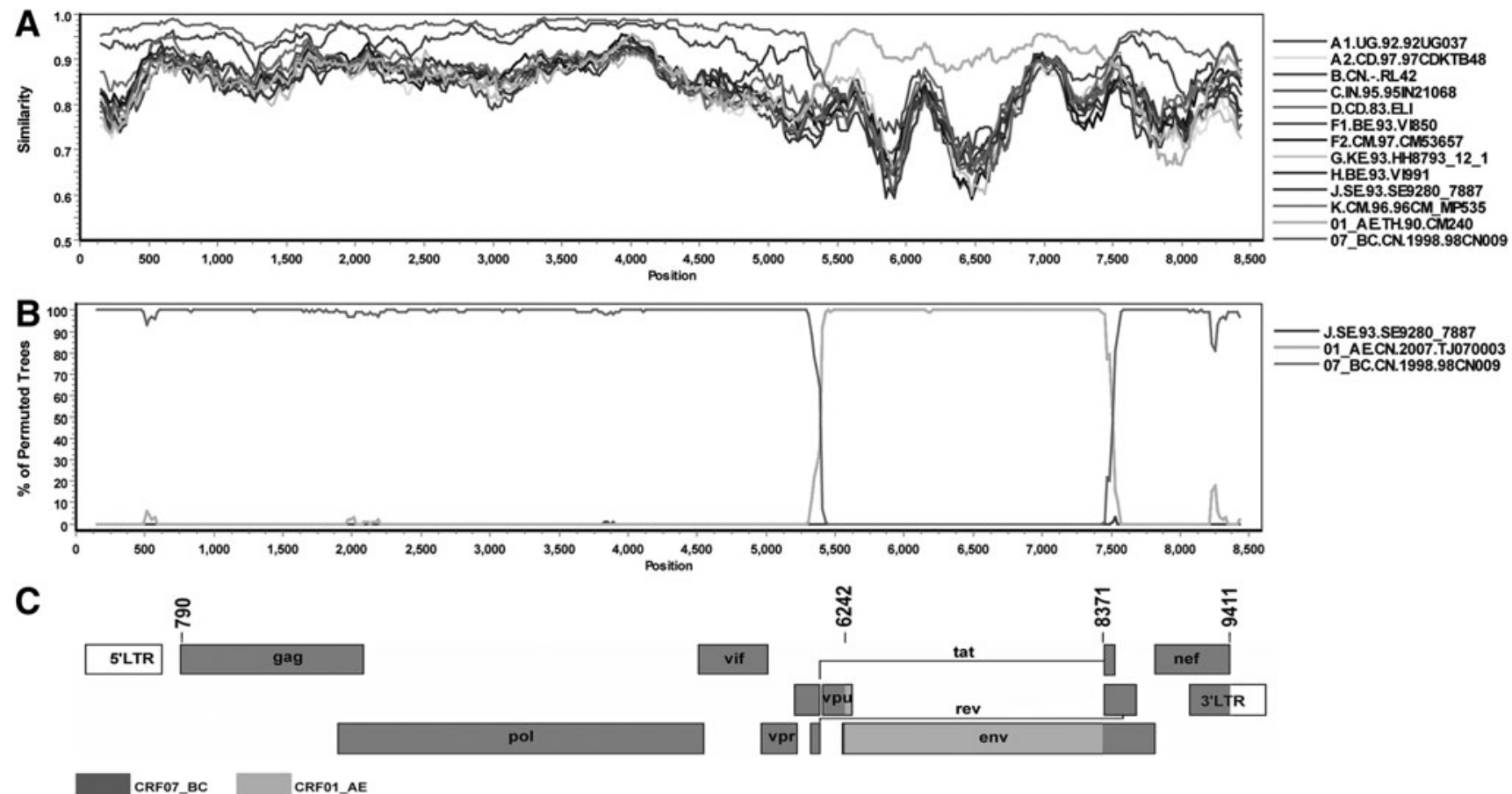

FIG. 2. (A) The similarity between JL.RF09 and the reference sequences was plotted using SimPlot 3.5.1 software. (B) Bootscanning analyses of the near full-length genome (NFLG) of JL.RF09. CRF01_AE (TJ070003) and CRF07_BC (98CN009) are used as putative parental reference sequences, and subtype J (SE9280_7887) is used as an outgroup. (C) The recombinant map results of JL.RF09. The schematic structure was created using the Recombinant HIV-1 Drawing Tool (www.hiv.lanl.gov/content/sequence/DRAW_CRF/recom_mapper.html).

The CRF07_BC regions (I and III) in JL.RF09 clustered with the CRF07_BC reference sequences (bootstrap value $100 \%$ ), but formed a distinct monophyletic branch between CRF07_BC Cluster 1 (designated CRF07-1) and other temporarily ungrouped CRF07_BC reference sequences. ${ }^{14-16} \mathrm{~A}$ potential reason for this could be that the parental origin of the CRF07_BC regions (I and III) was from a variant of CRF07_BC that NFLG has not characterized. In summary, the parental origins of the CRF01_AE and CRF07_BC regions of the novel second-generation recombinant (JL.RF09) could be from CRF01-4a and an unidentified CRF07_BC variant, respectively. This theory should be considered with caution until it is possible to obtain the full-length genome or NFLG sequences of the new CRF07_BC variant as a reference to indicate the real parental origin of CRF07_BC in this recombinant form. Therefore, it is necessary to increase the number of NFLG sequences of CRF07_BC to identify and better elucidate the transmission networks of the new CRF07_BC variant in this region.

To date, two NFLG sequences of HIV-1 CRF01_AE/ CRF07_BC second-generation recombinants (JL070032 and JL.RF09) composed of CRF01_AE and CRF07_BC have been detected in Jilin. The first reported HIV-1 CRF01_AE/ CRF07_BC unique recombinant (JL070032) in Jilin
I (nt 790-6241)

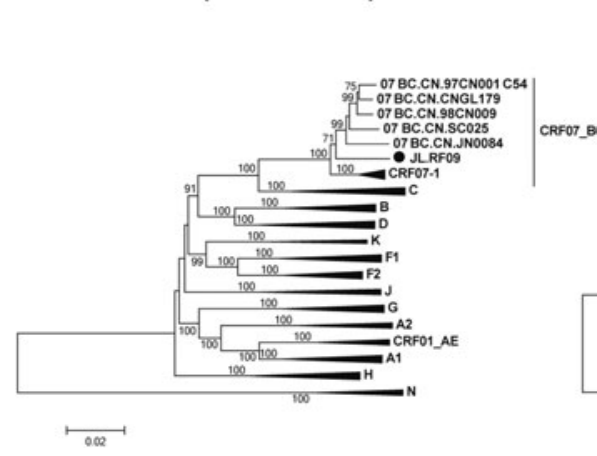

\section{II (nt 6242-8370)}

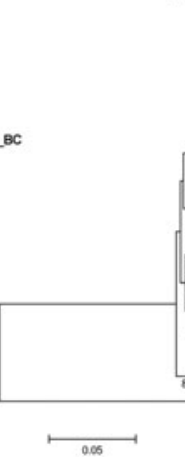

III (nt 8371-9411)

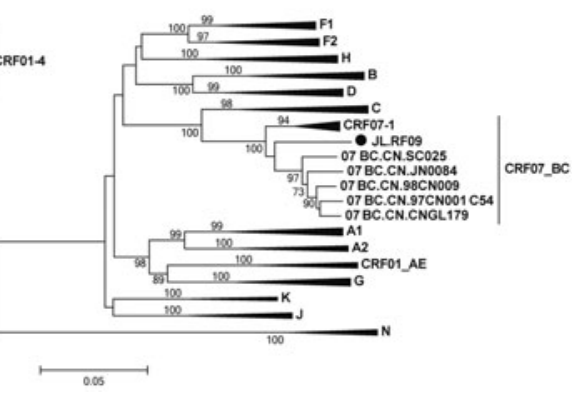

FIG. 3. The phylogenetic trees of subregions constructed by the neighbor-joining method with bootstrap values of $70 \%$ or greater indicated at the corresponding nodes. Reference sequences for the seven unique CRF01_AE lineages detected in China are labeled CRF01-1 through CRF01-7 and the Central African CRF01_AE sequences are labeled CRF01-CF. Reference sequences for the subcluster CRF07_BC lineage identified among men who have sex with men are labeled CRF07-1. 
province was identified from an HIV-positive consenting MSM subject. ${ }^{10}$ JL070032 has a complex recombinant structure with at least four breakpoints; two CRF07_BC regions were inserted into a backbone of CRF01_AE. The parental origin of the CRF01_AE regions of JL070032 was from CRF01_AE Cluster 5 (CRF01-5), which is another cluster of CRF01_AE mainly circulating among MSM in northern China.

The difference between the compositions of the two novel CRF01_AE/CRF07_BC recombinants reveals that they are unrelated recombinant variants. We also characterized other NFLG sequences of HIV-1 recombinants (CRF61_BC, CRF01_AE/B, and CRF01_AE/B'/C) from our previous study in this region. All of the recombinants are identified among heterosexuals or MSM. ${ }^{12,18-20}$ The emergence of multiple second-generation recombinants suggests the presence of complex transmission networks of different HIV subtype/CRF infections among sexual transmissions in this region. Therefore, a continuing HIV-1 molecular epidemiologic investigation is necessary to characterize the transmission networks within subtypes and CRFs, which is of great importance in understanding the dynamics of the HIV-1 epidemic in this region.

\section{Sequence Data}

The NLFG sequence of JL.RF09 has been deposited in GenBank under accession number KJ184180.

\section{Acknowledgments}

This study was supported by National Science and Technology Major Projects for Infectious Diseases Control and Prevention (2008ZX10001-004, 2012ZX10001-002, and 2012ZX10001-008). Additional support was provided by the National Natural Science Foundation of China (81261120379), by an International Cooperative grant (2009DFB30420), and by a SKLID Development grant (2012SKLID103).

\section{Author Disclosure Statement}

No competing financial interests exist.

\section{References}

1. Hemelaar J: The origin and diversity of the HIV-1 pandemic. Trends Mol Med 2012;18(3):182-192.

2. McCutchan FE: Understanding the genetic diversity of HIV-1. AIDS 2000;14(Suppl 3):S31-44.

3. He X, Xing H, Ruan Y, et al.: A comprehensive mapping of HIV-1 genotypes in various risk groups and regions across China based on a nationwide molecular epidemiologic survey. PLoS One 2012;7(10):e47289.

4. Chen J, Young NL, Subbarao S, et al.: HIV type 1 subtypes in Guangxi Province, China, 1996. AIDS Res Hum Retroviruses 1999;15(1):81-84.

5. Liao H, Tee KK, Hase $\mathrm{S}$, et al.: Phylodynamic analysis of the dissemination of HIV-1 CRF01_AE in Vietnam. Virology 2009;391(1):51-56.

6. Xing H, Liang H, Wan ZY, et al.: [Distribution of recombinant human immunodeficiency virus type-1 CRF01_ $\mathrm{AE}$ strains in China and its sequence variations in the env V3-C3 region]. Zhonghua Yu Fang Yi Xue Za Zhi 2004; 38(5):300-304.
7. Xing H, Pan PL, Su L, et al.: [Molecular epidemiological study of an HIV-1 strain of subtype E in China between 1996 and 1998]. Zhongguo Xing Bing Ai Zi Bing Fang Zhi 2002;8(4):200-203.

8. Meng Z, Xin R, Zhong P, et al.: A new migration map of HIV-1 CRF07_BC in China: Analysis of sequences from 12 provinces over a decade. PLoS One 2012;7(12):e52373.

9. Guo H, Guo D, Wei JF, et al.: First detection of a novel HIV Type 1 CRF01_AE/07_BC recombinant among an epidemiologically linked cohort of IDUs in Jiangsu, China. AIDS Res Hum Retroviruses 2009;25(4):463-467.

10. Li X, Ning C, He X, et al.: Near full-length genome sequence of a novel HIV type 1 second-generation recombinant form (CRF01_AE/CRF07_BC) identified among men who have sex with men in Jilin, China. AIDS Res Hum Retroviruses 2013;29(12):1604-1608.

11. Wei H, Su L, Feng Y, et al.: Near full-length genomic characterization of a novel HIV type 1 CRF07_BC/01_AE recombinant in men who have sex with men from Sichuan, China. AIDS Res Hum Retroviruses 2013;29(8):1173-1176.

12. Li $X$, Ning $C$, He $X$, et al.: Genome sequences of a novel HIV-1 circulating recombinant form (CRF61_BC) identified among heterosexuals in China. Genome Announc 2013;1(3).

13. Feng $\mathrm{Y}, \mathrm{He} \mathrm{X}$, Hsi $\mathrm{JH}$, et al.: The rapidly expanding CRF01_AE epidemic in China is driven by multiple lineages of HIV-1 viruses introduced in the 1990s. AIDS 2013;27(11):1793-1802.

14. Han X, Dai D, Zhao B, et al.: Genetic and epidemiologic characterization of HIV-1 infection in Liaoning Province, China. J Acquir Immune Defic Syndr 2010;53(Suppl 1): S27-33.

15. Li L, Lu X, Li H, et al.: High genetic diversity of HIV-1 was found in men who have sex with men in Shijiazhuang, China. Infect Genet Evol 2011;11(6):1487-1492.

16. Wang W, Xu J, Jiang S, et al.: The dynamic face of HIV-1 subtypes among men who have sex with men in Beijing, China. Curr HIV Res 2011;9(2):136-139.

17. Zhang L, Zhang D, Yu B, et al.: Prevalence of HIV infection and associated risk factors among men who have sex with men (MSM) in Harbin, P. R. China. PLoS One 2013;8(3):e58440.

18. Ning $\mathrm{C}, \mathrm{Li} X, \mathrm{He} X$, et al.: Near full-length genome identification of a novel HIV type $1 \mathrm{~B}^{\prime} / \mathrm{C}$ recombinant isolate JL100091 in Jilin, China. AIDS Res Hum Retroviruses 2013;29(12):1609-1612.

19. Li X, Ning C, Chen Y, et al.: Near full-length genome identification of a novel HIV-1 recombinant form (CRF01_AE/B'/C) among heterosexuals in Jilin, China. AIDS Res Hum Retroviruses 2014;30(7):695-700.

20. Li X, Feng Y, Yang Y, et al.: Near full-length genome sequence of a novel hiv-1 recombinant form (CRF01_AE/ B) detected among men who have sex with men in Jilin Province, China. AIDS Res Hum Retroviruses 2014;30(7): 701-705.

Address correspondence to: Yiming Shao

Division of Research on Virology and Immunology National Center for AIDS/STD Control and Prevention

China $C D C$

Beijing 102206

China

E-mail: yshao@bbn.cn 